\title{
Adaptive Image Resizing Based on Continuous-Domain Stochastic Modeling
}

\author{
Hagai Kirshner, Member, IEEE, Aurélien Bourquard, Student Member, IEEE, John Paul Ward, \\ Moshe Porat, Senior Member, IEEE, and Michael Unser, Fellow, IEEE
}

\begin{abstract}
We introduce an adaptive continuous-domain modeling approach to texture and natural images. The continuous-domain image is assumed to be a smooth function, and we embed it in a parameterized Sobolev space. We point out a link between Sobolev spaces and stochastic auto-regressive models, and exploit it for optimally choosing Sobolev parameters from available pixel values. To this aim, we use exact continuousto-discrete mapping of the auto-regressive model that is based on symmetric exponential splines. The mapping is computationally efficient, and we exploit it for maximizing an approximated Gaussian likelihood function. We account for non-Gaussian Lévytype processes by deriving a more robust estimator that is based on the sample auto-correlation sequence. Both estimators use multiple initialization values for overcoming the local minima structure of the fitting criteria. Experimental image resizing results indicate that the auto-correlation criterion can cope better with non-Gaussian processes and model mismatch. Our work demonstrates the importance of the auto-correlation function in adaptive image interpolation and image modeling tasks, and we believe it is instrumental in other image processing tasks as well.
\end{abstract}

Index Terms-Auto-regressive parameter estimation, adaptive interpolation, exponential splines.

\section{INTRODUCTION}

$\mathbf{I}$ MAGE modeling is fundamental to many image processing tasks such as resizing, restoration, analysis and compression. With the increasing use of various visual monitors of different resolution characteristics and the handy options of changing the size of the displayed image, resizing has become a common task in image processing. The underlying idea of currently available linear resizing methods is a regularity constraint that is imposed on the continuousdomain image. Polynomial B-spline modeling is such an example for which pixel values correspond to the samples of a piecewise polynomial function. Once a continuous-domain model is determined, one can sample it on the required new

Manuscript received January 31, 2013; revised September 8, 2013; accepted September 23, 2013. Date of publication October 17, 2013; date of current version November 28, 2013. This work was supported in part by the Swiss National Science Foundation under Grant 200020-121763, in part by the European Commission under Grant ERC- 2010-AdG 267439-FUN-SP, and in part by the Ollendorff Minerva Center. Minerva is funded through the BMBF. The associate editor coordinating the review of this manuscript and approving it for publication was Prof. Aleksandra Pizurica.

H. Kirshner, A. Bourquard, J. P. Ward, and M. Unser are with the Biomedical Imaging Group, École Polytechnique Fédérale de Lausanne, Lausanne CH-1015, Switzerland (e-mail: hagai.kirshner@epfl.ch; aurelien.bourquard@epfl.ch; john.ward@epfl.ch; michael.unser@epfl.ch).

M. Porat is with the Department of Electrical Engineering, Technion-Israel Institute of Technology, Haifa 32000, Israel (e-mail: mp@ee.technion.ac.il).

Color versions of one or more of the figures in this paper are available online at http://ieeexplore.ieee.org.

Digital Object Identifier 10.1109/TIP.2013.2285597 grid or algebraically project it onto a new function space. Both theoretical and experimental studies have shown that the B-spline kernels provide an excellent trade-off in terms of interpolation performance and computational complexity [1], [2]. Nonlinear methods, on the other hand, rely on local properties of edges [3]-[5], on statistical properties of wavelet coefficients [6]-[8], on Markov random field stochastic models [9], [10], and on regularized inverse problem formulations of differential type [11]-[13], to name a few. The main advantage of these methods resides in their ability to locally adapt the interpolation weights to the given data. The drawback, however, is computational complexity.

The question we are raising in this work is whether one can benefit from these two types of methods. Namely, introduce a fast linear interpolation method that adapts itself to the given data. We are in particularly interested in a modeling approach that would be invariant to the pixel size value. That is, if two snapshots of the same image are acquired at different pixel size values, we require the adapted continuous-domain model to be the same for both.

We introduce in this work an adaptive continuous-domain modeling approach to texture and natural images. The continuous-domain image is assumed to be a smooth function, and we embed it in a parametrized Sobolev space. We then point out a link between Sobolev spaces and stochastic auto-regressive models, and exploit it for optimally choosing Sobolev parameters from available pixel values. To this aim, we use exact continuous-to-discrete mapping of the auto-regressive model that is based on symmetric exponential splines. The mapping is computationally efficient, and we exploit it for maximizing an approximated Gaussian likelihood function. We further account for non-Gaussian Lévytype processes by deriving an estimator that is based on the sample auto-correlation values only. Both of our estimators use multiple initialization values for overcoming the local minima structure of the fitting criteria. Auto-correlation values are useful for describing texture images [14]. While the available models are formulated in the discrete-domain, our approach is based on the continuum. Exceptions to that are the continuous-domain formulations [15] and interpolation [16], [17] by means of an adapted auto-correlation function. We take this idea one step further by accounting for autoregressive models that can take arbitrary order values and that can have non-Gaussian innovations.

The advantage of the proposed model resides in its ability to describe both sparse and non-sparse signals, while maintaining a linear forward model. Unlike piecewise 


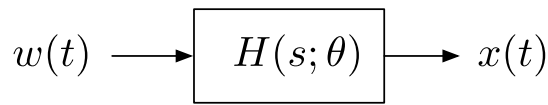

Fig. 1. A description of a continuous-domain AR process: $w(x)$ is a continuous-domain white noise (Gaussian or non-Gaussian), and $H(s ; \theta)$ is an all pole causal filter, given in the Laplace domain.

polynomial models, the Sobolev space parametrization introduces flexibility in determining the auto-correlation properties of the digital image. The Lévy innovation model is particularly interesting from a signal processing point of view. It covers the whole range of infinitely divisible probability density functions, which guarantees the existence of a probability density function of the available uniformly sampled data. We demonstrate the usefulness of our approach by considering the task of image resizing, and further compare its performance with the polynomial B-spline kernel.

The paper is organized as follows. In Section II, we provide the link between continuous-domain Lévy-type AR processes and Sobolev functions. We then present a continuous-todiscrete mapping of the AR parameters. In Section III, we introduce two estimators from sampled data. One estimator uses the Gaussian likelihood function, and the other estimator is based on a more general setup. Experimental results are described in Section IV.

\section{The Proposed Model}

\section{A. Auto-Regressive Processes With Lévy-Type Innovation}

We consider a general continuous-domain $\operatorname{AR}(p)$ model with parameters vector $\theta$ (Figure 1)

$$
v(x)=\int_{0}^{\infty} h(x-\xi ; \theta) w(\xi) d \xi,
$$

where $w(x)$ is a Gaussian or non-Gaussian white-noise process. The equivalent stochastic-integral representation is

$$
v(x)=\int_{0}^{\infty} h(x-\xi ; \theta) d W(\xi),
$$

where $W(x)$ is a Brownian motion (in the Gaussian case) or a more general Lévy process (in the non-Gaussian case) [18]. The connection between both representations is

$$
W(x)=\int_{0}^{x} w(\xi) d \xi .
$$

Conversely, $w(x)=\frac{d}{d x} W(x)$, which may be termed white Lévy noise or innovation, is a generalized stochastic process that corresponds to the derivative of $W(x)$ in the sense of distributions [19]. Sparsity is determined by the infinitesimal increments of $W(x)$ (Figure 2). In the case of a Brownian motion, the increments are Gaussian random variables, and $v(x)$ has no sparse innovation. In the Poisson case, however, a large portion of the increments are zero, which results in a sparse innovation [20].

The shaping filter $h(x ; \theta)$ is given in the Fourier domain by

$$
H(\omega ; \theta)=\frac{1}{\prod_{n=1}^{p}\left(i \omega-s_{n}\right)},
$$

where

$$
\theta=\left(s_{1}, s_{2}, \ldots, s_{p}\right) \in \mathbb{C}^{p} .
$$

The vector of parameters $\theta$ consists of the poles of $H(\omega ; \theta)$. The real part of each pole is strictly negative, and complex poles appear in conjugate pairs. We Assume that $w(x)$ is white noise with finite variance $\sigma^{2}$. Then, for sufficiently large values of $x$, the autocorrelation function $\varphi(\xi)=\mathbf{E}\{v(x) \cdot v(x+\xi)\}$ is given in the Fourier domain by

$$
\Phi\left(\omega ; \theta, \sigma^{2}\right)=\sigma^{2}|H(\omega ; \theta)|^{2}=\frac{\sigma^{2}}{\prod_{n=1}^{p}\left(i \omega-s_{n}\right)\left(-i \omega-s_{n}\right)} .
$$

The advantage of the proposed model resides in its ability to describe both sparse and non-sparse signals, while maintaining a linear forward model. The parametrization $\theta$ introduces flexibility in determining the auto-correlation properties of $v(x)$, as well. The Lévy innovation model covers the whole range of infinitely divisible probability density functions, which are particularly interesting from a signal processing point of view. The infinite divisibility property guarantees the existence of a probability density function of the available uniformly sampled data.

\section{B. The Link With Deterministic Signals}

Let $\mathcal{H}_{2}(\lambda)$ be a Sobolev space with scalar weights $\lambda=\left\{\lambda_{n}\right\}_{0}^{p}$. This space consists of all finite-energy functions for which their first $p$ derivatives are of finite energy, as well. The Sobolev inner product is given by

$$
\langle x, y\rangle_{\mathcal{H}_{2}(\lambda)}=\sum_{n=0}^{p} \lambda_{n} \cdot\left\langle x^{(n)}, y^{(n)}\right\rangle_{L_{2}},
$$

where $\lambda$ provides a positive measure for $\langle x, x\rangle_{\mathcal{H}_{2}(\lambda)}$. The reproducing kernel of $\mathcal{H}_{2}(\lambda)$ is the unique function satisfying

$$
x(\tau)=\langle x(t), \varphi(t-\tau ; \lambda)\rangle_{\mathcal{H}_{2}(\lambda)},
$$

for every $x \in \mathcal{H}_{2}(\lambda)$ and $\tau \in \mathbb{R}$. It is given in the Laplace domain by [21]

$$
\Phi(s ; \lambda)=\frac{1}{\lambda_{0}-\lambda_{1} s^{2}+\cdots+(-1)^{p} \cdot \lambda_{p} s^{2 p}} .
$$

Considering a uniform grid, the sample values of a Sobolev function uniquely define its orthogonal projection, with respect to the Sobolev norm, onto the subspace

$$
\mathcal{S}(\lambda)=\overline{\operatorname{span}\{\varphi(\mathrm{t}-\mathrm{n} ; \lambda)\}_{\mathrm{n} \in \mathbb{Z}}} .
$$

Both $x$ and its orthogonal projection $P_{\mathcal{S}(\lambda)} x$ have the same sample values. The orthogonal projection is also the solution to the following interpolation problem [21]

$$
\left\{P_{\mathcal{S}(\lambda)} x\right\}(\tau)=\underset{\alpha \in \mathbb{R}}{\operatorname{argmin}} \max _{\|y\|<\infty,\{y(n T)=x(n T)\}}|y(\tau)-\alpha|,
$$

where the norms are taken in the Sobolev sense. Interpolated values can be calculated in practice by

$$
\left\{P_{\mathcal{S}(\lambda)} x\right\}(\tau)=u^{T} G^{-1} v,
$$

where $u[n]=x(n T)$ are the known sample values, $v[n]=$ $\varphi(n T-\tau ; \lambda)$ are point-wise values of the reproducing kernel, 


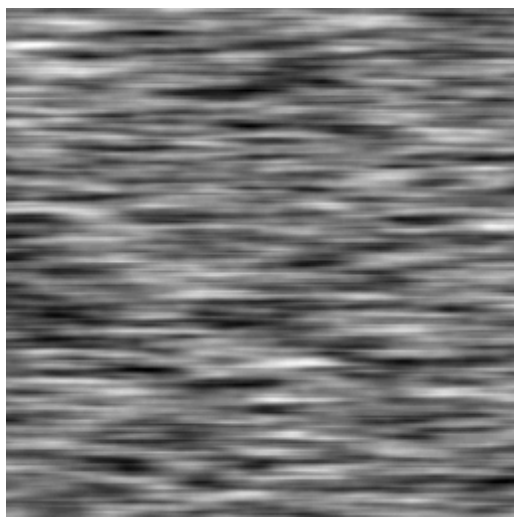

(a)

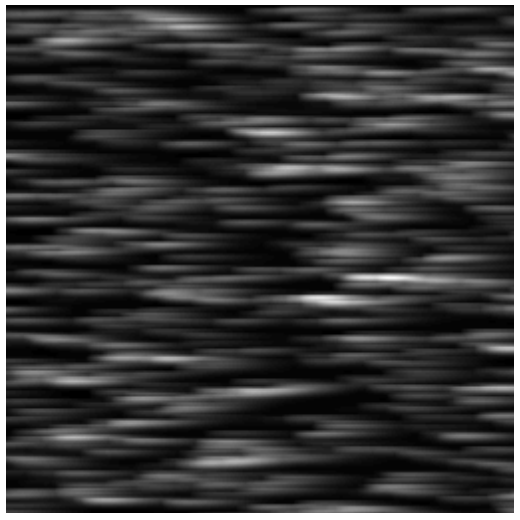

(c)

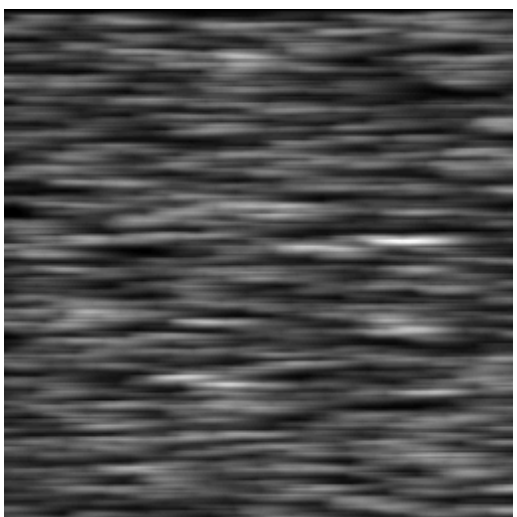

(b)

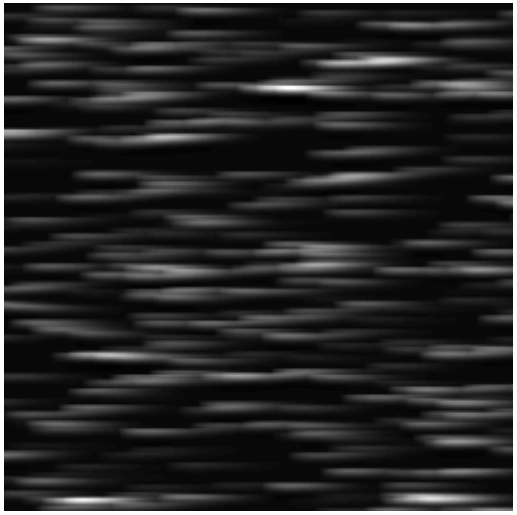

(d)

Fig. 2. Examples of sparse and non-sparse stochastic processes: Shown here are pixel values of a 2D continuous-domain AR process $v(x)$. The shaping filter in the horizontal direction has two complex poles $-0.05 \pm 0.05 i$, and the poles in the vertical direction are $-0.5 \pm 0.5 i$. The non-sparse image (a) is based on a continuous-domain Gaussian innovation process. The images (b)-(d) are based on continuous-domain Poisson process with different average density values $-1 / 20,1 / 80,1 / 160$. The size of each image is $256 \times 256$ pixels, the sampling interval is of a unit value, and the integral step size in (1) is 0.2 .

and $G(m, n)=\varphi((m-n) T ; \lambda)$ is the Gram matrix of the functions in (9).

This deterministic modeling is closely related to stochastic AR processes. In particular, the Laplace transform (9) describes an auto-regressive auto-correlation function, which has the same role as the reproducing kernel in signal interpolation. The linear minimum mean squared error (LMMSE) estimator of the random variable $x(\tau)$ coincides with the deterministic value $P_{\mathcal{S}(\lambda)} x(\tau)$ when setting the two functions to be equal [21].

The equivalence between deterministic Sobolev modeling and stochastic AR processes can be utilized to adaptively determine the weights $\lambda$ from the available sample values. We suggest here to estimate continuous-domain AR parameters from pixel values; determine the power spectrum of the underlying continuous-domain process; and extract the corresponding Sobolev weights.

\section{Continuous-to-Discrete Mapping}

In order to estimate the continuous-domain parameters $\theta$ of a given image, we first characterize the stochastic properties of sampled continuous-domain AR models. Upon sampling, such models become discrete-domain auto-regressive moving average (ARMA) signals [22]. This means that the sampled data can be statistically modeled by a discrete-domain uncorrelated input signal that undergoes a digital filtering operation (Figure 3). The digital filter has a rational transfer function in the $z$-transform domain, and we express its poles and zeros by means of a symmetric exponential spline $\beta(t ; \theta)$. We first introduce its Laplace transform and then define the ARMA digital filter $H_{d}(z ; \theta)$. The latter will then be used to define its inverse filter $G_{d}(z ; \theta)$ and a likelihood term $\kappa(\theta)$; both will be shown to compose a maximum likelihood objective function (in the Gaussian case) and an $l_{2}$ error criterion (in the non-Gaussian case).

Definition 1: The localization filter of $\theta$ is

$$
\Delta(s ; \theta)=\prod_{k=1}^{p}\left(1-e^{s+s_{k}}\right)\left(1-e^{-s+s_{k}}\right) .
$$

Definition 2: The symmetric exponential B-spline of $\theta$ is given in the Laplace domain by

$$
\mathcal{B}(s ; \theta)=\Delta(s ; \theta) \cdot \Phi(s ; \theta) .
$$

The $z$ transform of its sampled version is

$$
\mathcal{B}_{d}(z ; \theta)=\sum_{n=-(p-1)}^{p-1} \beta(n ; \theta) z^{-n} .
$$




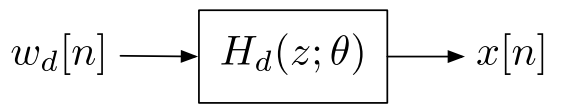

Fig. 3. Sampling a continuous-time AR process: the sampled process can be described by a discrete-time innovation $w_{d}[n]$ that undergoes a digital filtering operation. The digital filter $H_{d}(z ; \theta)$ has a rational transfer function, and the time-series is therefore an ARMA model.

In the Gaussian case, the discrete-time innovation is i.i.d; in the non-Gaussian case, it is uncorrelated only. We define next $H_{d}(z: \theta), G_{d}(z ; \theta)$ and $\kappa(\theta)$; they will be used later on for parameters estimation.

Definition 3: The discrete-time ARMA parameters that correspond to the sampled version of the continuous-time process $\theta$ are [22]

$$
H_{d}(z ; \theta)=\frac{\prod_{k=1}^{p-1}\left(1-v_{k}(\theta) z^{-1}\right)}{\prod_{k=1}^{p}\left(1-\rho_{k}(\theta) z^{-1}\right)},
$$

where

$$
\begin{aligned}
& \rho_{k}(\theta)=e^{s_{k}} \\
& v_{k}(\theta)=\text { roots of } \mathcal{B}_{d}(z ; \theta) \text { inside the unit circle. }
\end{aligned}
$$

The variance of the innovation is

$$
\sigma_{d}^{2}(\theta)=\sigma^{2} \frac{\mathcal{B}_{d}(1 ; \theta)}{\prod_{k=1}^{p-1}\left(1-v_{k}(\theta)\right)^{2}} .
$$

Definition 4: Let $\theta$ be known. Then,

$$
G_{d}(z ; \theta)=1 / H_{d}(z ; \theta)
$$

Definition 5: Let $\theta$ be known. Then,

$$
\kappa(\theta)=\sum_{n=1}^{\infty} n \cdot c[n ; \theta]^{2}
$$

where

$$
c[n ; \theta]=\frac{1}{n}\left\{v_{1}^{n}(\theta)+\cdots+v_{p-1}^{n}(\theta)-\rho_{1}^{n}(\theta)-\cdots-\rho_{p}^{n}(\theta)\right\} .
$$

\section{PARAmeters Estimation From PiXel VAlues}

In this section we use the $1 \mathrm{D}$ digital filter $G_{d}(z ; \theta)$ and the term $\kappa(\theta)$ to derive a likelihood function $l\left(\theta_{x}, \theta_{y} ; x\right)$ and an $l_{2}$ fitting criterion $\epsilon_{x}^{2}\left(\theta_{x}\right), \epsilon_{y}^{2}\left(\theta_{y}\right)$ for a given image. To this aim, we assume a separable two-dimensional $\operatorname{AR}(p)$ model

$$
\Phi\left(s_{x}, s_{y} ; \theta\right)=\Phi_{x}\left(s_{x} ; \theta_{x}\right) \cdot \Phi_{y}\left(s_{y} ; \theta_{y}\right)
$$

where $\Phi_{x}, \Phi_{y}$ are $1 \mathrm{D}$ continuous-domain $\operatorname{AR}(p)$ processes. The vector of parameters is

$$
\theta=(\sigma^{2}, \underbrace{s_{1, x}, \ldots, s_{p, x}}_{\mathrm{x} \text { direction }}, \underbrace{s_{1, y}, \ldots, s_{p, y}}_{\mathrm{y} \text { direction }}),
$$

where $\sigma^{2}=\sigma_{x}^{2} \sigma_{y}^{2}$.
Definition 6: The row-ordering lexicographic representation of an $M$ rows by $N$ columns image $x$ is

$$
\begin{aligned}
\tilde{x}= & (\underbrace{x[0,0], \ldots, x[0, N-1]}_{\text {First row }}, \underbrace{x[1,0], \ldots, x[1, N-1]}_{\text {Second row }}, \\
& \ldots, \underbrace{x[M-1,0], \ldots, x[M-1, N-1]}_{\text {Last row }}) .
\end{aligned}
$$

Definition 7: The auto-covariance matrix of $\tilde{x}$ is given by

$$
\Sigma(k, l ; \theta)=\varphi_{x}\left(\tilde{k} T ; \theta_{x}\right) \cdot \varphi_{y}\left(\tilde{l} T ; \theta_{y}\right),
$$

where $\tilde{k}=(k \bmod N)-(l \bmod N)$ and $\tilde{l}=(k \operatorname{rem} N)-$ $(l \operatorname{rem} N)$.

\section{A. Fitting Criteria}

\section{1) Brownian Motion-The Gaussian Case:}

Definition 8: The log-likelihood function of $x$ is

$$
l(\theta ; x)=\ln |\Sigma(\theta)|+\tilde{x}^{T}(\Sigma(\theta))^{-1} \tilde{x},
$$

Definition 9: The approximated likelihood function of $x$ is

$$
\begin{aligned}
\tilde{l}(\theta ; x)= & M \kappa\left(\theta_{x}\right)+N \kappa\left(\theta_{y}\right)+M^{2} \ln \sigma_{d}^{2}\left(\theta_{x}\right) \\
& +N^{2} \ln \sigma_{d}^{2}\left(\theta_{y}\right)+\left\|x * \mathbf{g}_{\theta}\right\|_{\ell_{2}}^{2},
\end{aligned}
$$

where $g_{\theta}[m, n]=g_{\theta_{x}}[m] * g_{\theta_{y}}[n]$ and $*$ denotes a $2 \mathrm{D}$ convolution operation.

The approximated likelihood function $\tilde{l}(\theta ; x)$ converges uniformly in $\theta$ to $l(\theta ; x)$ with an increasing number of samples

$$
\lim _{\min (M, N) \rightarrow \infty} \mathbf{E}_{x}|l(\theta ; x)-\tilde{l}(\theta ; x)|=0 .
$$

This stems from the block structure of the auto-correlation matrix $\Sigma(\theta)=\Sigma_{y}\left(\theta_{y}\right) \otimes \Sigma_{x}\left(\theta_{x}\right)$, where $\otimes$ denotes Kronecker product. It then holds that $|\Sigma(\theta)|=\left|\Sigma_{y}\left(\theta_{y}\right)\right|^{M}\left|\Sigma_{x}\left(\theta_{x}\right)\right|^{N}$, and from [22] we have that

$$
\lim _{M \rightarrow \infty}\left\{\ln \left|\Sigma_{x}\left(\theta_{x}\right)\right|-\kappa\left(\theta_{x}\right)-M \ln \sigma_{d}^{2}\left(\theta_{x}\right)\right\}=0 .
$$

The 2D whitening operation in (27) is separable, and we observe that

$$
\Sigma(\theta)^{-1}=\Sigma_{y}\left(\theta_{y}\right)^{-1} \otimes \Sigma_{x}\left(\theta_{x}\right)^{-1} .
$$

This means that the AR image can be whitened by applying column- and row-wise filtering operations, and we use

$$
\lim _{\min (M, N) \rightarrow 0} \mathbf{E}_{x}|| \tilde{x}^{T}(\Sigma(\theta))^{-1} \tilde{x}\left|-\left\|x * \mathbf{g}_{\theta_{x}} * \mathbf{g}_{\theta_{y}}\right\|^{2}\right|=0
$$

which holds true due to the asymptotic equivalence of $\Sigma_{x}\left(\theta_{x}\right)$ and $\Sigma_{y}\left(\theta_{y}\right)$ with $\mathbf{g}_{\theta_{x}}$ and $\mathbf{g}_{\theta_{y}}$, respectively.

The uniform convergence (29) implies that the global minimum of $\tilde{l}(\theta ; x)$ provides a reasonable approximation of the global minimum of $l(\theta ; x)$. The advantage of minimizing the former resides in its low computational complexity; it involves the parameters mapping (16) and the convolution operation $x * g_{\theta}$ only. 
TABLE I

Parameter Estimation from PiXel Values-Validation of the Proposed Approach

\begin{tabular}{|c|c|c|c|c|c|c|c|c|c|}
\hline \multirow[t]{3}{*}{ Lévy-type innovation } & \multirow[t]{3}{*}{$\sigma^{2}$} & \multirow[t]{3}{*}{ Poles } & \multirow[t]{3}{*}{$a, b$} & \multicolumn{6}{|c|}{ Estimation Error (relative values) } \\
\hline & & & & \multicolumn{3}{|c|}{ Gaussian likelihood } & \multicolumn{3}{|c|}{ autocorrelation fit } \\
\hline & & & & $\sigma^{2}$ & $a$ & $b$ & $\sigma^{2}$ & $a$ & $b$ \\
\hline \multirow{4}{*}{ Gaussian } & \multirow{2}{*}{1} & $x:-1 \pm 1 i$ & $(2,2)$ & \multirow{2}{*}{$<0.0001$} & 0.0009 & 0.0007 & \multirow{2}{*}{0.007} & 0.0006 & 0.0004 \\
\hline & & $y:-2 \pm 1 i$ & $(4,5)$ & & 0.013 & 0.014 & & 0.008 & 0.009 \\
\hline & \multirow{2}{*}{1} & $x:-1 \pm 1 i$ & $(2,2)$ & \multirow{2}{*}{$<0.0001$} & 0.0006 & 0.0005 & \multirow{2}{*}{0.007} & 0.003 & 0.002 \\
\hline & & $y:-1 \pm 5 i$ & $(2,26)$ & & 0.0001 & 0.0014 & & 0.0006 & 0.0008 \\
\hline \multirow{4}{*}{ Poisson } & \multirow{2}{*}{1} & $x:-1 \pm 1 i$ & $(2,2)$ & \multirow{2}{*}{2.2} & 0.9 & 0.1 & \multirow{2}{*}{0.4} & 0.006 & 0.002 \\
\hline & & $y:-2 \pm 1 i$ & $(4,5)$ & & 1.7 & 244.7 & & 0.008 & 0.007 \\
\hline & \multirow{2}{*}{1} & $x:-1 \pm 1 i$ & $(2,2)$ & \multirow{2}{*}{0.7} & 0.1 & 0.01 & \multirow{2}{*}{0.4} & 0.001 & 0.0005 \\
\hline & & $y:-1 \pm 5 i$ & $(2,26)$ & & 0.7 & 15.3 & & 0.0008 & 0.0015 \\
\hline \multirow{4}{*}{ Poisson } & \multirow{2}{*}{$1 / 80$} & $x:-1 \pm 1 i$ & $(2,2)$ & \multirow{2}{*}{0.005} & 0.0007 & 0.0008 & \multirow{2}{*}{0.0001} & 0.005 & 0.003 \\
\hline & & $y:-2 \pm 1 i$ & $(4,5)$ & & 0.02 & 0.02 & & 0.008 & 0.008 \\
\hline & \multirow{2}{*}{$1 / 80$} & $x:-1 \pm 1 i$ & $(2,2)$ & \multirow{2}{*}{0.005} & 0.002 & 0.002 & \multirow{2}{*}{$<0.0001$} & 0.002 & 0.001 \\
\hline & & $y:-1 \pm 5 i$ & $(2,26)$ & & 0.0008 & 0.002 & & 0.0004 & 0.001 \\
\hline
\end{tabular}

2) Non-Gaussian Scenario: When $W(t)$ is not Gaussian, the mapping of (16) still holds true, but the discrete-domain innovation is no longer Gaussian. Rather, it presents a Markov-type dependency between its values, and the probabilistic properties of its values depend on the Lévy parameters of $W(t)$. Instead of deriving explicit likelihood expressions for each Lévytype innovation, we suggest to fit the sample auto-correlation sequence of the image with a continuous-domain AR model. In particular, separately minimizing the following $l_{2}$ error criteria

$$
\begin{aligned}
\epsilon_{x}^{2}\left(\theta_{x}\right) & =\sum_{k=0}^{K}\left|\tilde{\varphi}(k, 0)-\varphi\left(k ; \theta_{x}\right)\right|^{2} \\
\epsilon_{y}^{2}\left(\theta_{y}\right) & =\sum_{l=0}^{L}\left|\tilde{\varphi}(0, l)-\varphi\left(l ; \theta_{y}\right)\right|^{2},
\end{aligned}
$$

where

$$
\begin{aligned}
\tilde{\varphi}(k, l)= & \frac{1}{(M-k-1)(N-l-1)} \\
& \cdot \sum_{m=0}^{M-l-1} \sum_{n=0}^{N-k-1}[x(m, n)-\bar{x}][x(m+k, n+l)-\bar{x}],
\end{aligned}
$$

and $\bar{x}$ is the average value of $x$. The parameters $K, L$ specify how many auto-correlation sample values are taken into account for the estimation.

\section{B. Multiple Minimizations}

Both the Gaussian approximated likelihood function $\tilde{l}(\theta ; x)$ and the error criteria $\epsilon_{x}^{2}\left(\theta_{x}\right), \epsilon_{y}^{2}\left(\theta_{y}\right)$ have local minima. In order to find their global minimum, we suggest to use multiple minimizations with different initialization values. The main idea is to set the values of $\theta_{x}$ and $\theta_{y}$ sufficiently close to one of the local minima. This can be achieved by estimating the decay rate of the sample auto-correlation.
1) Decay Rate Estimation: We fit the values of $\log \tilde{\varphi}(k, 0)$ with a regression line; the slope of the line, denoted $\alpha$, indicates the slowest decay rate of the auto-correlation function along the $x$ direction. The number of values that are used for the regression are provided by the user. We apply a similar regression for the $y$ direction.

2) $A R(1)$ : We use a single initialization for each direction

$$
\begin{aligned}
s_{1} & =\alpha \\
\sigma^{2} & =\tilde{\varphi}(0,0)\left[\frac{\mathcal{B}_{d}\left(1 ; s_{1}\right)}{\prod_{k=1}^{p-1}\left(1-v_{k}\left(s_{1}\right)\right)^{2}}\right]^{-1} .
\end{aligned}
$$

3) $A R(2)$ : We use multiple initializations for each direction $\left\{\left(s_{1_{k}}, s_{2_{k}}\right)\right\}_{k \in \mathbb{N}^{0}}$ where

$$
\left(s_{1_{k}}, s_{2_{k}}\right)= \begin{cases}(\alpha, \alpha / 2) & k=0 \\ (\alpha+k i, \alpha-k i) & \text { else }\end{cases}
$$

The first initialization values, i.e. $k=0$, corresponds to two real poles. The other initialization conditions correspond to two complex conjugate poles. The number of complex poles is provided by the user. For each pair of poles, we compute

$$
\sigma^{2}=\tilde{\varphi}(0,0)\left[\frac{\mathcal{B}_{d}\left(1 ; s_{1}, s_{2}\right)}{\prod_{k=1}^{p-1}\left(1-v_{k}\left(s_{1}, s_{2}\right)\right)^{2}}\right]^{-1} .
$$

4) $A R(3)$ : We use multiple initializations for each direction $\left\{\left(s_{1_{k}}, s_{2_{k}}, s_{3_{k}}\right)\right\}_{k \in \mathbb{N} 0}$ where

$$
\left(s_{1_{k}}, s_{2_{k}}, s_{3_{k}}\right)= \begin{cases}(\alpha, \alpha / 2, \alpha / 3) & k=0 \\ (\alpha+k i, \alpha-k i, \alpha / 2) & \text { else }\end{cases}
$$

For each triplet of poles, we compute

$$
\sigma^{2}=\tilde{\varphi}(0,0)\left[\frac{\mathcal{B}_{d}\left(1 ; s_{1}, s_{2}, s_{3}\right)}{\prod_{k=1}^{p-1}\left(1-v_{k}\left(s_{1}, s_{2}, s_{3}\right)\right)^{2}}\right]^{-1} .
$$




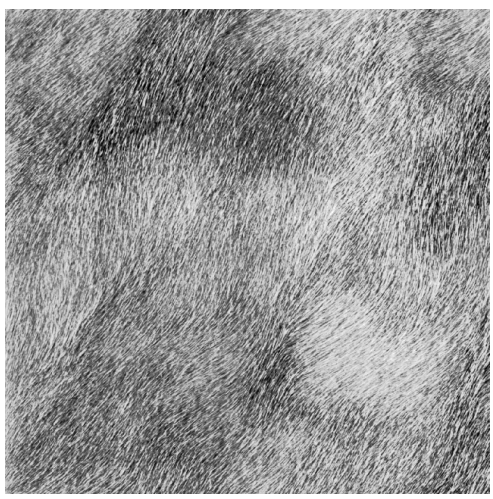

(a)

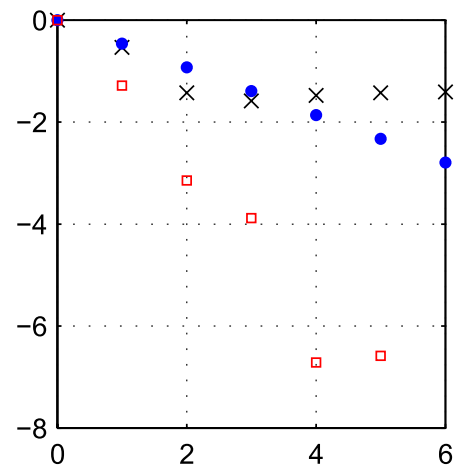

(b)

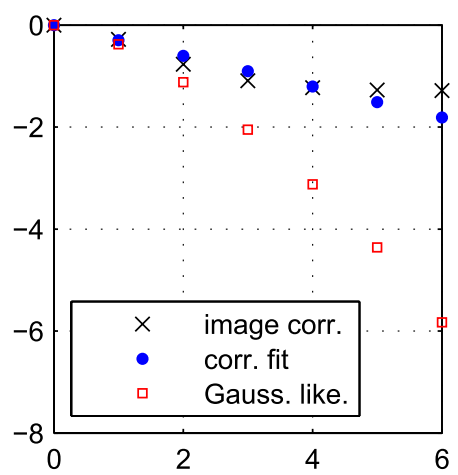

(c)

Fig. 4. Validation of the proposed estimation approach: shown in (a) is the D93 Brodatz texture image. The sample auto-correlation sequences along the $x$ and $y$ directions are shown in (b) and (c) by $\times$ marks. Auto-correlation values that were obtained by minimizing the Gaussian likelihood function are depicted by squares; the values that were obtained by minimizing the auto-correlation fit criterion are shown by circles. This example demonstrates the advantage of the auto-correlation error criterion for adapting a continuous-domain AR model to an image with an unknown Lévy innovation.

5) General $A R(p)$ : The initialization values need to cover all possible constellations of real and complex poles. The real part of the first pole is $\alpha$. The real part of any other pole is either equal to the real part of its predecessor (if it is its complex conjugate) or smaller than its predecessor (if it is real). The imaginary part of a complex pair can take multiple values, namely $k i$ where $k \in \mathbb{N}^{0}$.

\section{Validation}

Table I presents estimation results for simulated images. The data corresponds to pixel values of Gaussian and non-Gaussian continuous-domain two-dimensional $\operatorname{AR}(2)$ processes. The estimated parameters are $\left(\sigma^{2}, a, b\right)$, where $a, b$ are the polynomial coefficients of the shaping filter

$$
\begin{aligned}
H(\omega ; \theta) & =\frac{1}{\left(i \omega-s_{1}\right)\left(i \omega-s_{2}\right)} \\
& =\frac{1}{-\omega^{2} \underbrace{-\left(s_{1}+s_{2}\right)}_{a} i \omega+\underbrace{s_{1} s_{2}}_{b}} .
\end{aligned}
$$

Our Monte-Carlo simulation included 20 realizations for each AR model, and the error criterion was the mean squared error. The values that appear in the table are normalized by the oracle values of each model. The size of the images was $256 \times 256$. As expected, the Gaussian likelihood estimator performs well for Gaussian images. However, it is less suited to other types of innovations. For Poisson-driven images, the estimation performance depends on the variance value $\sigma^{2}$. If it is small, then the Gaussian likelihood value is dominated by the norm of the discrete-domain innovation, which amounts to whitening the data by the correct parameters. When the value of $\sigma^{2}$ is dominating the likelihood value, the estimation performance becomes worse. This is due to the fact that we need to restrict the maximum value of $\sigma^{2}$ in the numerical optimization. The auto-correlation fit criterion, on the other hand, performs well for both Gaussian and non-Gaussian images. It is therefore more robust to modeling inaccuracies. This property is further illustrated in Figure 4.

\section{EXPERIMENTAL RESULTS}

We demonstrate the usefulness of our modeling approach by considering the task of image resizing. The estimated AR parameters determine a Sobolev space, and we resize the image by sampling $P_{\mathcal{S}(\lambda)} x$ on the new grid. The compactlysupported exponential B-spline of Definition 2 is instrumental for deriving a computationally efficient reconstruction procedure. It introduces the same computational complexity as the polynomial B-spline kernel of [23], [24]. In particular,

$$
P_{\mathcal{S}(\lambda)} x(\eta, \zeta)=\sum_{m=0}^{M-1} \sum_{n=0}^{N-1} c[m, n] \beta\left(\eta-n ; \theta_{x}\right) \beta\left(\zeta-m ; \theta_{y}\right),
$$

where $c$ is given in the $z$-transform domain by

$$
C\left(z_{x}, z_{y}\right)=X\left(z_{x}, z_{y}\right) \frac{1}{\mathcal{B}_{d}\left(z_{x} ; \theta_{x}\right)} \frac{1}{\mathcal{B}_{d}\left(z_{y} ; \theta_{y}\right)} .
$$

In Tables II and III, we present resizing results of simulated AR images, texture, ${ }^{1}$ and natural images. The quality of the resized images is evaluated both in terms of SNR and in terms of the structural-similarity-index measure ${ }^{2}$ (SSIM) of [26].

The original image was down-sampled by a factor of two (Table II) and three (Table III) in each dimension while applying no averaging pre-filter. Continuous-domain AR parameters were estimated from the down-sampled image. These parameters were then used in (43) for resizing the image back to its original size. Both the Gaussian likelihood estimator and the auto-correlation estimator are reported in the tables; the auto-correlation criterion performed better in all cases. The number of auto-correlation values that were used in estimating the decay rate was $K=L=5$, and the maximum

\footnotetext{
${ }^{1}$ The texture images were taken from the Brodatz database at http://www.ux.uis.no/ tranden/brodatz.html and from the database http://www-cvr.ai.uiuc.edu/ponce_grp/data/ that was used in [25].

${ }^{2}$ The SSIM measure aims at being consistent with perceived visual quality. Each given SSIM value is a real number defined between 0 and 1 . We use a MATLAB implementation available at https://ece.uwaterloo.ca/ z70wang/research/ssim/.
} 
TABLE II

IMAGE RESIZING By A FACTOR OF TWO IN EACH DiMENSION

\begin{tabular}{|c|c|c|c|c|c|c|}
\hline \multirow[t]{2}{*}{ Original Image } & \multicolumn{3}{|c|}{ SNR $[\mathrm{dB}]$} & \multicolumn{3}{|c|}{ SSIM } \\
\hline & Polynomial & $\begin{array}{l}\text { Exponential } \\
\text { (auto-corr.) }\end{array}$ & $\begin{array}{l}\text { Exponential } \\
\text { (likelihood) }\end{array}$ & Polynomial & $\begin{array}{l}\text { Exponential } \\
\text { (auto-corr.) }\end{array}$ & $\begin{array}{l}\text { Exponential } \\
\text { (likelihood) }\end{array}$ \\
\hline$A R(1024 \times 1024)^{*}$ & 6.3 & 6.5 & 6.5 & 0.926 & 0.927 & 0.927 \\
\hline$D 2(640 \times 640)$ & 19.2 & 19.3 & 6.2 & 0.940 & 0.938 & 0.196 \\
\hline D79 $(640 \times 640)$ & 12.3 & 12.6 & 5.3 & 0.870 & 0.873 & 0.153 \\
\hline D93 $(640 \times 640)$ & 15.5 & 15.7 & 9.0 & 0.849 & 0.820 & 0.143 \\
\hline$D 105(640 \times 640)$ & 5.1 & 5.2 & 2.9 & 0.410 & 0.413 & 0.085 \\
\hline T04_01 $(640 \times 480)$ & 20.1 & 20.8 & 19.3 & 0.800 & 0.820 & 0.804 \\
\hline T04_26 $(640 \times 480)$ & 20.9 & 21.5 & 10.3 & 0.857 & 0.872 & 0.248 \\
\hline T15_30 $(640 \times 480)$ & 19.1 & 19.7 & 10.2 & 0.836 & 0.856 & 0.485 \\
\hline T20_01 $(640 \times 480)$ & 8.6 & 9.0 & 4.7 & 0.770 & 0.796 & 0.679 \\
\hline$T 22 \_16(640 \times 480)$ & 16.9 & 17.4 & 14.7 & 0.886 & 0.887 & 0.831 \\
\hline Couple $(512 \times 512)$ & 23.0 & 23.2 & 23.1 & 0.940 & 0.942 & 0.941 \\
\hline Fingerprint $(512 \times 512)$ & 27.1 & 27.2 & 27.1 & 0.990 & 0.990 & 0.990 \\
\hline Hill $(512 \times 512)$ & 23.8 & 24.3 & 24.2 & 0.936 & 0.941 & 0.940 \\
\hline Man $(512 \times 512)$ & 23.9 & 24.0 & 23.7 & 0.953 & 0.954 & 0.952 \\
\hline Pentagon $(1024 \times 1024)$ & 23.4 & 23.7 & 12.2 & 0.975 & 0.977 & 0.284 \\
\hline
\end{tabular}

* Simulated $\mathrm{AR}(2)$ process with Gaussian innovation and poles $-0.8 \pm i$ and $-0.2 \pm 0.6 i$ in $x$ and $y$, respectively.

imaginary-part value considered in the search space was $2 \pi$. The AR order was $p=2$. To conform with the model, the DC components of the down-sampled images were subtracted before the estimation and interpolation operations and added back to the corresponding up-scaled results. We also report resizing results that were obtained by the cubic polynomial B-spline kernel; no average subtraction is required in this case due to the partition-of-unity property of this kernel. This kernel is compactly supported between $[-2,2]$, as is the exponential B-spline kernel of our approach when $p=2$. Both kernels have the same approximation order, as well. Visual comparisons between the proposed adaptive model and the polynomial B-spline model are given in Figures 5 and 6 for texture-image and natural-image examples, respectively. Similar results are shown in Figure 7 for an instance where upscaling only is performed on an image crop. Note that, in this latter case, the data are provided as such and the oracle solution is thus unavailable.
The results of the tables show that our adaptive approach introduces notable improvements of up to $0.7[\mathrm{~dB}]$ over the non-adaptive polynomial spline model when considering a single resizing operation. The SSIM values are also consistent with these SNR improvements, which indicates that the perceived visual quality also increases. More accurate details can indeed be observed in the visual results specific to our method. For instance, the Brodatz texture D79 (top row) that is reconstructed using our approach contains vertical patterns that are more pronounced and closer to the original version compared to the non-adaptive case. Moreover, the images we obtain look sharper than the piecewise polynomial model, indicating that our adaptive approach can overcomes aliasing. The AR model introduces a parameterized family of power spectrum functions $\Phi(\omega ; \theta)$ which can be of either lowpass or bandpass nature. Our multiple minimization algorithm examines these power spectra in an efficient manner, and extracts the parameters that best fit the given data. The corresponding 
TABLE III

IMAGE RESIZING B Y A FACTOR OF THREE IN EACH DIMENSION

\begin{tabular}{|c|c|c|c|c|c|c|}
\hline \multirow[t]{2}{*}{ Original Image } & \multicolumn{3}{|c|}{ SNR $[\mathrm{dB}]$} & \multicolumn{3}{|c|}{ SSIM } \\
\hline & Polynomial & $\begin{array}{l}\text { Exponential } \\
\text { (auto-corr.) }\end{array}$ & $\begin{array}{l}\text { Exponential } \\
\text { (likelihood) }\end{array}$ & Polynomial & $\begin{array}{l}\text { Exponential } \\
\text { (auto-corr.) }\end{array}$ & $\begin{array}{l}\text { Exponential } \\
\text { (likelihood) }\end{array}$ \\
\hline$A R(1024 \times 1024)^{*}$ & 2.2 & 3.0 & 3.0 & 0.711 & 0.731 & 0.729 \\
\hline D2 $(640 \times 640)$ & 16.0 & 16.4 & 7.8 & 0.942 & 0.945 & 0.118 \\
\hline D79 $(640 \times 640)$ & 9.1 & 9.7 & 6.5 & 0.827 & 0.852 & 0.216 \\
\hline D93 $(640 \times 640)$ & 12.4 & 13.1 & 10.1 & 0.824 & 0.839 & 0.251 \\
\hline D105 $(640 \times 640)$ & 4.5 & 4.6 & 4.0 & 0.309 & 0.313 & 0.138 \\
\hline T04_01 $(640 \times 480)$ & 18.6 & 19.3 & 11.8 & 0.656 & 0.684 & 0.167 \\
\hline T04_26 $(640 \times 480)$ & 19.2 & 19.9 & 11.3 & 0.721 & 0.740 & 0.170 \\
\hline T15_30 $(640 \times 480)$ & 17.3 & 18.1 & 11.1 & 0.703 & 0.739 & 0.359 \\
\hline T20_01 $(640 \times 480)$ & 5.8 & 6.5 & 3.4 & 0.536 & 0.602 & 0.443 \\
\hline$T 22 \_16(640 \times 480)$ & 14.1 & 14.8 & 10.6 & 0.718 & 0.728 & 0.480 \\
\hline Couple $(512 \times 512)$ & 19.4 & 19.8 & 7.8 & 0.844 & 0.853 & 0.272 \\
\hline Fingerprint $(512 \times 512)$ & 21.7 & 21.8 & 7.5 & 0.952 & 0.951 & 0.098 \\
\hline Hill $(512 \times 512)$ & 21.1 & 21.5 & 21.5 & 0.856 & 0.865 & 0.863 \\
\hline Man $(512 \times 512)$ & 20.7 & 21.0 & 6.7 & 0.875 & 0.881 & 0.150 \\
\hline Pentagon $(1024 \times 1024)$ & 20.6 & 21.3 & 13.7 & 0.915 & 0.927 & 0.220 \\
\hline
\end{tabular}

${ }^{*}$ Simulated $\mathrm{AR}(2)$ process with Gaussian innovation and poles $-0.8 \pm i$ and $-0.2 \pm 0.6 i$ in $x$ and $y$, respectively.

power spectrum may then occupy frequency bins that are not necessarily in the baseband. This is the key point of our algorithm in overcoming aliasing that is introduced by the downsampled image. A similar observation can be made in Figure 7 even though no oracle is available for comparison in such a case.

The results of Tables II and III further indicate that the autocorrelation fit estimator performs well for all images, whereas the Gaussian likelihood estimator yields strongly suboptimal solutions except for the simulated AR image. The reason for that is the numerical limits we imposed on the innovation variance $\sigma^{2}$ during the optimization process. The tables show that the auto-correlation fit is a general estimator for the continuous-domain AR model that does not depend on the type of innovation and that can be applied satisfactorily to practical visual data.

The performance of our approach depends on the extent to which an image complies with the AR model, and on the amount of aliasing introduced by the downsampled image. This observation is motivated by the relatively large improvement of the SNR measure for the simulated AR image in Table III. If the downsampled image introduces prominent aliasing, such as in a downsampling factor of three, then it is more likely to have a significant improvement by the proposed algorithm which can cope with oscillatory structures. This is the case in Table III for the $D 2(0.4[\mathrm{~dB}])$ and T04_1 (0.7 [dB]) images. The image T15_30 (0.8 [dB]) is not oscillatory, though. Rather, it introduces fine details and sharp transitions that are captured by a bandpass AR model whereas the piecewise polynomial model cannot accommodate for such frequencies. From a computational perspective, our method is equivalent to the polynomial spline model; the only overhead is the adaptive parameter-estimation stage, which introduces only marginal additional complexity.

Resizing results for standard test-sets natural images are presented in the tables, too. Even though such images are not necessarily stationary, as implicitly assumed by our model, our approach remains advantageous compared to the piecewisepolynomial model in most cases. This observation is illustrated by the visual example of Figure 6 . The estimated 

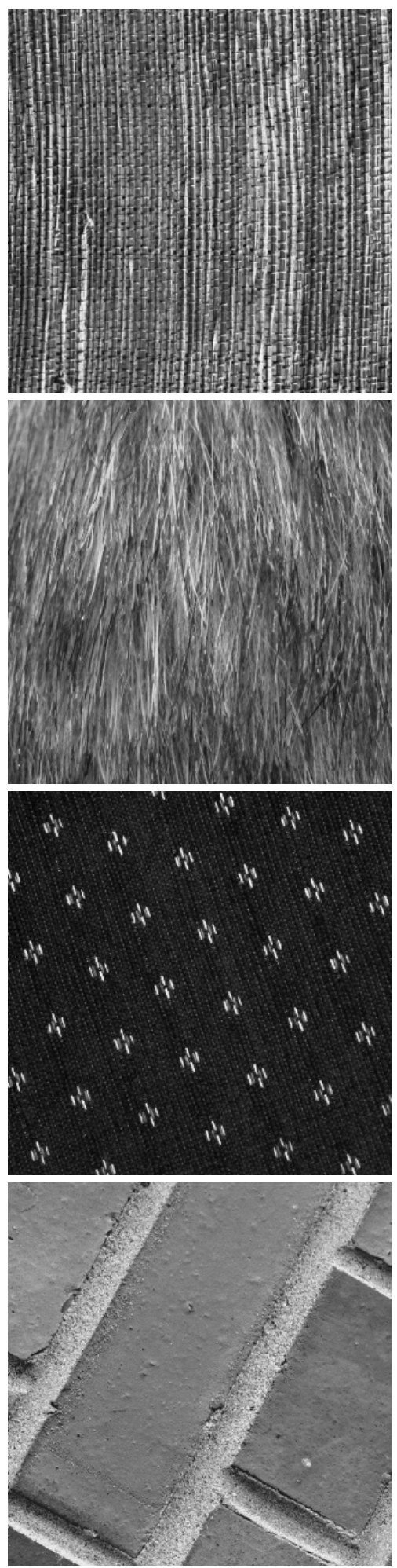

Original
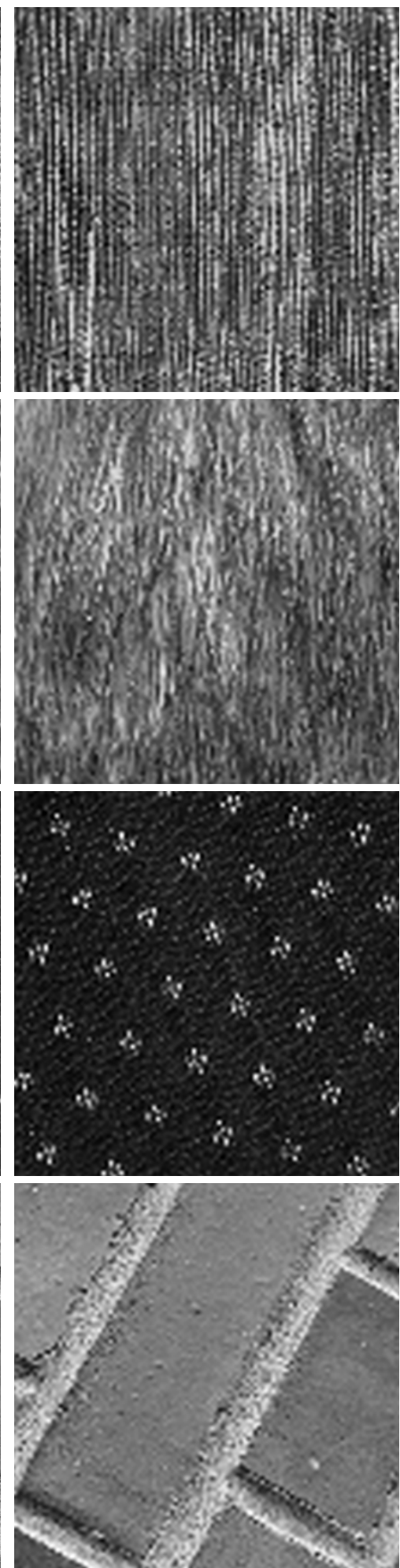

Non-adaptive (polynomial)
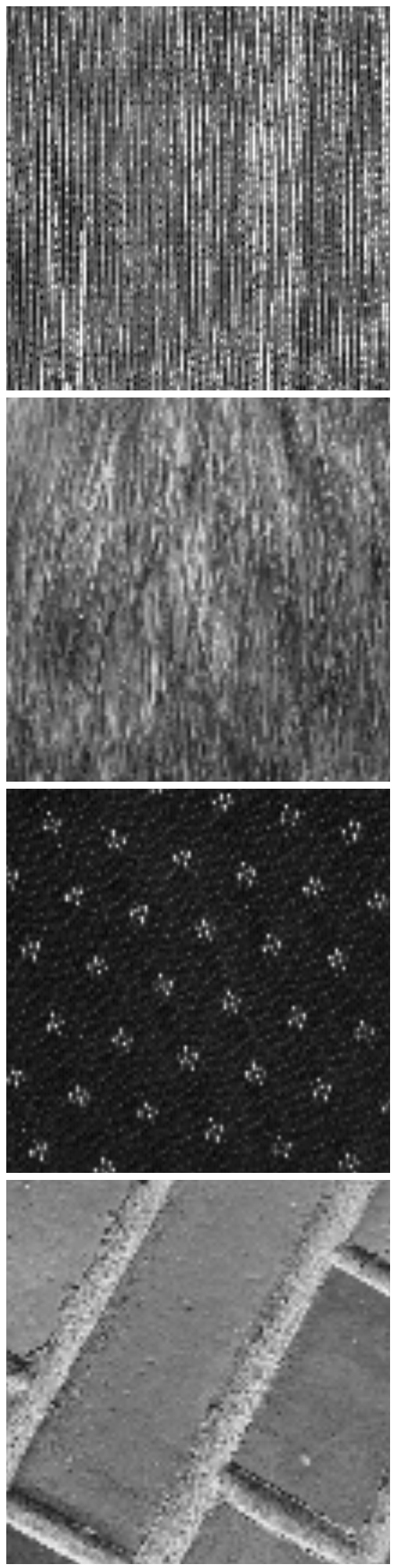

Adaptive (proposed)

Fig. 5. Image resizing results: the original image (left column) was down-sampled by a factor of three in each dimension. Resizing was carried out by the polynomial B-spline model (middle column) and by the proposed adaptive approach (right column). The images are D79 (top row), T22_16 (second row), T20_01 (third row), and T15_30 (bottom row). Shown here are centered crops of size $320 \times 320$ pixels.

parameters in this configuration can be interpreted as an average model evaluated on the different stationary regions of the image. The corresponding quality improvements demonstrate the important role that the auto-correlation function has in image interpolation.
Our experimental results indicate that the proposed approach is essentially resolution-invariant. We correctly identified continuous-domain AR parameters from simulated images that introduce prominent aliasing effects in the power spectrum function due to the down-sampling operation. 


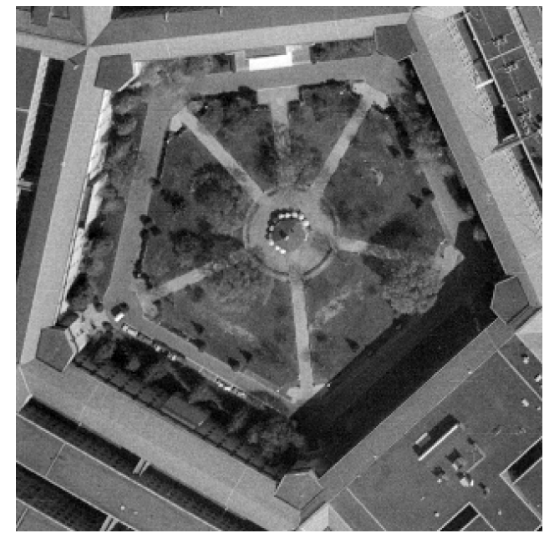

Original

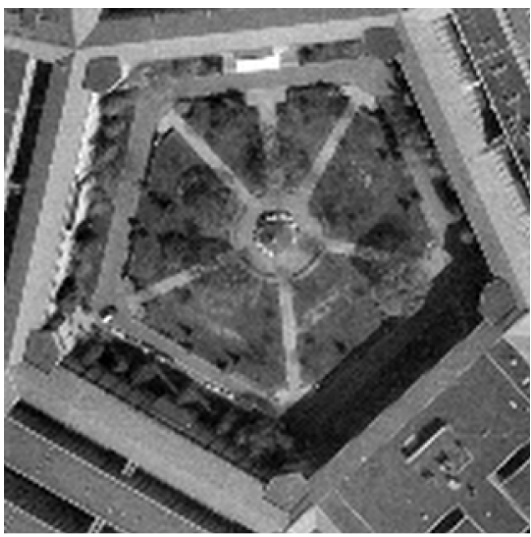

Non-adaptive (polynomial)

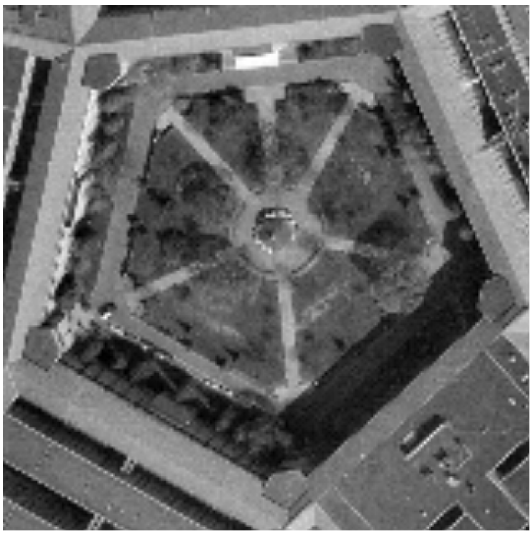

Adaptive (proposed)

Fig. 6. Image resizing results: the Pentagon image (left) was down-sampled by a factor of two in each dimension. Resizing was carried out by the cubic polynomial B-spline model (middle) and by the proposed adaptive approach (right). Shown is a crop of size $320 \times 320$ pixels encompassing the central shape.

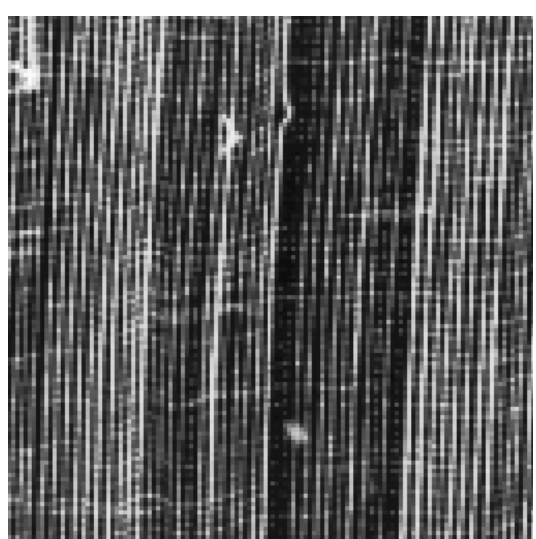

Original

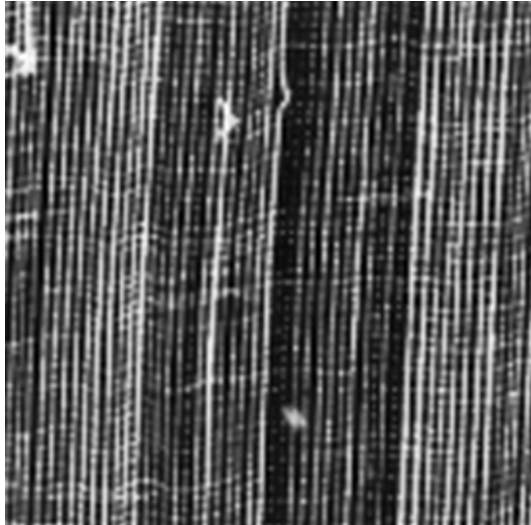

Non-adaptive (polynomial)

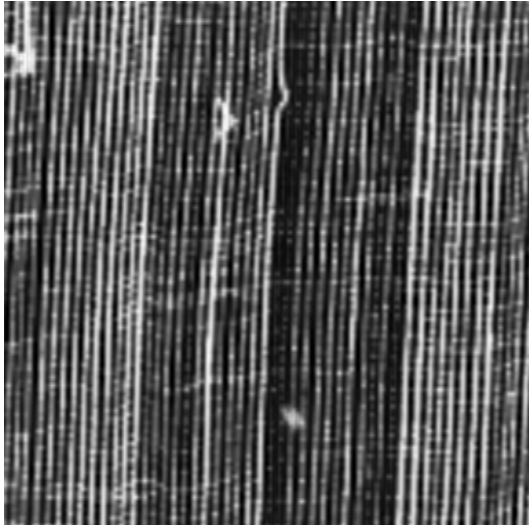

Adaptive (proposed)

Fig. 7. Image resizing results: the data consists of a $128 \times 128$ crop of $D 105$. Resizing by a factor of three in each dimension was carried out by the cubic polynomial B-spline model (middle) and by the proposed adaptive approach (right). The original data (left) is shown at the same scale as the resized images using pixel duplication.

Moreover, we achieved improved interpolation results for down-sampled texture and natural images. The proposed estimation approach relies on the uniqueness results of $\operatorname{AR}(2)$ processes [27], which states that the sampling interval value imposes no constraint on the one-to-one mapping between the continuous- and the discrete domains AR models. Similar uniqueness results hold for AR models of higher orders, which raises the question of optimal model order selection. One possible way of choosing the optimal order for a given image is by minimizing the $l_{2}$ approximation errors $\epsilon_{x}^{2}\left(\theta_{x}\right)$ and $\epsilon_{y}^{2}\left(\theta_{y}\right)$ using several values of the model order $p$. That is, for every possible value of $p$, we find the best parameters $\theta_{x}, \theta_{y}$. We then choose the model order that yields the minimum error values.

\section{CONCLUSION}

We introduced in this work an adaptive, yet computationally efficient, linear resizing approach for digital images. We described the sampling operator by means of an orthogonal projection in a continuous-domain Soblev space, and optimized the Sobolev weights to the given image. This was carried out by relating the Sobolev reproducing kernel function with a continuous-domain auto-regressive model. Pixel values were assumed to be a realization of such a process, and we used them to estimate the auto-regressive parameters. We derived a maximum-likelihood estimator for the Gaussian case, and a more general estimator that accounts for the larger class of Lévy-type processes. The proposed estimators use multiple initializations for overcoming the aliasing effect in the down-sampled image. Our results also demonstrate that the Gaussian maximum-likelihood estimator is in many cases unsuitable for describing texture and natural images; the estimator that is based on the autocorrelation sample values provided a better alternative. It achieved higher SNR values, compared to the polynomial B-spline approach, while maintaining a linear image resizing formulation. We demonstrated the important role the auto-correlation measure has in image resizing, and we believe it is instrumental in other image processing tasks, as well.

\section{REFERENCES}

[1] T. Blu, P. Thévenaz, and M. Unser, "MOMS: Maximal-order interpolation of minimal support," IEEE Trans. Image Process., vol. 10, no. 7, pp. 1069-1080, Jul. 2001.

[2] E. H. W. Meijering, W. J. Niessen, and M. A. Viergever, "Quantitative evaluation of convolution-based methods for medical image interpolation," Med. Image Anal., vol. 5, no. 2, pp. 111-126, Jun. 2001. 
[3] K. Jensen and D. Anastassiou, "Subpixel edge localization and the interpolation of still images," IEEE Trans. Image Process., vol. 4, no. 3 , pp. 285-295, Mar. 1995.

[4] X. Li and M. Orchard, "New edge-directed interpolation," IEEE Trans. Image Process., vol. 10, no. 10, pp. 1521-1527, Oct. 2001.

[5] L. Zhang and X. Wu, "An edge-guided image interpolation algorithm via directional filtering and data fusion," IEEE Trans. Image Process., vol. 15 , no. 8, pp. 2226-2238, Aug. 2006.

[6] W. Carey, D. Chuang, and S. Hemami, "Regularity-preserving image interpolation," IEEE Trans. Image Process., vol. 8, no. 9, pp. 1293-1297, Sep. 1999.

[7] S. Chang, Z. Cvetkovic, and M. Vetterli, "Locally adaptive waveletbased image interpolation," IEEE Trans. Image Process., vol. 15, no. 6, pp. 1471-1485, Jun. 2006.

[8] J. Wang, S. Zhu, and Y. Gong, "Resolution-invariant image representation and its applications," in Proc. IEEE CVPR, Jun. 2009, pp. 2512-2519.

[9] X. Zhang and X. Wu, "Image interpolation by adaptive 2-D autoregressive modeling and soft-decision estimation," IEEE Trans. Image Process., vol. 17, no. 6, pp. 887-896, Jun. 2008.

[10] M. Li and T. Nguyen, "Markov random field model-based edge-directed image interpolation," IEEE Trans. Image Process., vol. 17, no. 7, pp. 1121-1128, Jul. 2008.

[11] F. Malgouyres and F. Guichard, "Edge direction preserving image zooming: A mathematical and numerical analysis," SIAM J. Numer. Anal., vol. 39, no. 1, pp. 1-37, 2001.

[12] T. F. Chan and J. Shen, Image Processing and Analysis: Variational, PDE, Wavelet, and Stochastic Methods. Philadelphia, PA, USA: SIAM, 2005.

[13] A. Bourquard, P. Thévenaz, K. Balać, and M. Unser, "Consistent and regularized magnification of images," in Proc. IEEE ICIP, Oct. 2008, pp. $325-328$.

[14] M. Petrou and P. G. Sevilla, Image Processing: Dealing with Texture. New York, NY, USA: Wiley, 2006.

[15] C.-L. Chang and B. Girod, "Image modeling with parametric texture sources for design and analysis of image processing algorithms," in Proc. VCIP, Jan. 2008, pp. 1-6.

[16] S. Nemirovski and M. Porat, "On texture and image interpolation using Markov models," Signal Process., Image Commun., vol. 24, pp. 139-157, May 2009.

[17] T. Chen and R. Defigueiredo, "Two-dimensional interpolation by generalized spline filters based on partial differential equation image models," IEEE Trans. Acoust., Speech, Signal Process., vol. 33, no. 3, pp. 631-642, Jun. 1985

[18] P. Protter, Stochastic Integration and Differential Equations. New York, NY, USA: Springer-Verlag, 2004.

[19] I. Gelfand and N. Y. Vilenkin, Generalized Functions (Applications of Harmonic Analysis), vol. 4. New York, NY, USA: Academic, 1964.

[20] M. Unser and P. D. Tafti, "Stochastic models for sparse and piecewise-smooth signals," IEEE Trans. Signal Process., vol. 59, no. 3, pp. 989-1006, Feb. 2011

[21] H. Kirshner and M. Porat, "On the role of exponential splines in image interpolation," IEEE Trans. Signal Process., vol. 18, no. 10, pp. 2198-2208, Oct. 2009.

[22] H. Kirshner, S. Maggio, and M. Unser, "A sampling theory approach to continuous ARMA identification," IEEE Trans. Signal Process., vol. 59, no. 10, pp. 4620-4634, Oct. 2011.

[23] P. Thévenaz, T. Blu, and M. Unser, "Interpolation revisited," IEEE Trans Med. Imaging, vol. 19, no. 7, pp. 739-758, Jul. 2000.

[24] M. Unser, A. Aldroubi, and M. Eden, "B-spline signal processing: Part I-Theory," IEEE Trans. Signal Process., vol. 41, no. 2, pp. 821-833, Feb. 1993.

[25] S. Lazebnik, C. Schmid, and J. Ponce, "A sparse texture representation using local affine regions," IEEE Trans. Pattern Anal. Mach. Intell., vol. 27 , no. 8 , pp. $1265-1278$, Aug. 2005 .

[26] Z. Wang, A. C. Bovik, H. R. Sheikh, and E. P. Simoncelli, "Image quality assessment: From error measurement to structural similarity," IEEE Trans. Image Process., vol. 13, no. 4, pp. 851-564, May 2004

[27] J. P. Ward, H. Kirshner, and M. Unser, "Is uniqueness lost for under-sampled continuous-time auto-regressive processes?" IEEE Signal Process. Lett., vol. 19, no. 4, pp. 183-186, Apr. 2012.

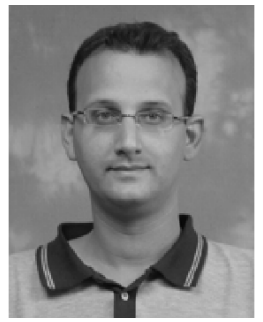

Hagai Kirshner received the B.Sc. (summa cum laude), M.Sc., and Ph.D. degrees in electrical engineering from the Technion-Israel Institute of Technology, Haifa, Israel, in 1997, 2005, and 2009, respectively. He is currently a Post-Doctoral Fellow at the Biomedical Imaging Group, École Polytechnique Fédérale de Lausanne, Lausanne, Switzerland. From 1997 to 2004, he was a System Engineer with the Technology Division, IDF. His research interests include sampling theory, stochastic image representation and processing, and computational methods in super-resolution microscopy.

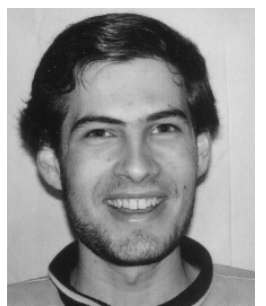

Aurélien Bourquard (M'08) received the M.Sc degree in microengineering from the Swiss Federal Institute of Technology (EPFL), Lausanne, Switzerland, in 2008. He is currently pursuing the Ph.D. degree with the Biomedical Imaging Group, EPFL. His research interests include image reconstruction using convex optimization and multigrid techniques, as well as new acquisition methods in the framework of computational optics.

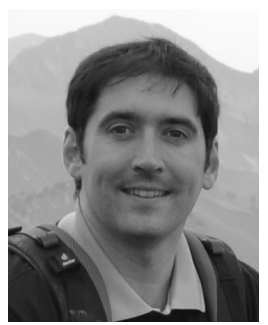

John Paul Ward received the B.S. degree in mathematics from the University of Georgia, Athens, GA, USA, and the Ph.D. degree in mathematics from Texas A\&M University, College Station, TX, USA, in 2005 and 2010, respectively. He is currently a Post-Doctoral Researcher with the Biomedical Imaging Group, Swiss Federal Institute of Technology, Lausanne, Switzerland.

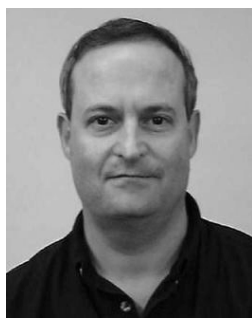

Moshe Porat (S'86-M'97-SM'97) received the B.Sc. (summa cum laude) and D.Sc. degrees in electrical engineering from the Technion - Israel Institute of Technology, Haifa, Israel, in 1982 and 1987, respectively. Since 1988, he has been on the faculty of electrical engineering Technion. During 1991-1992, he was with the Signal Processing Department, AT\&T Bell laboratories, Murray Hill, NJ, USA, on leave from the Technion. Dr. Porat has also held visiting positions at several universities, including the University of Auckland, Auckland, New Zealand, where he spent a sabbatical year from 2001 to 2002 as an Associate Professor at the Department of Electrical and Electronic Engineering. His professional interests are in the area of signal processing, specializing in localized representations of signals for image processing and computer vision.

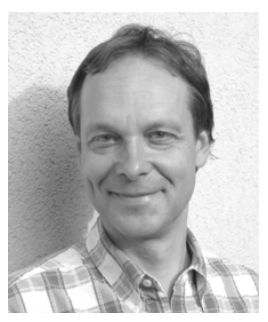

Michael Unser (F'99) is a Professor and the Director of EPFL's Biomedical Imaging Group, Lausanne, Switzerland. His main research area is biomedical image processing. He has a strong interest in sampling theories, multiresolution algorithms, wavelets, the use of splines for image processing, and, more recently, stochastic processes. He has published over 250 journal papers on those topics. From 1985 to 1997 , he was with the Biomedical Engineering and Instrumentation Program, National Institutes of Health, Bethesda, USA, conducting research on bioimaging and heading the Image Processing Group. He is a EURASIP fellow (2009), and a member of the Swiss Academy of Engineering Sciences. $\mathrm{He}$ is the recipient of several international prizes including three IEEE-SPS Best Paper Awards and two Technical Achievement Awards from the IEEE (2008 SPS and EMBS 2010). 